\title{
REVIEW
}

\section{Management of septic arthritis: a systematic review}

\author{
C J Mathews, G Kingsley, M Field, A Jones, V C Weston, M Phillips, D Walker, G Coakley
}

Ann Rheum Dis 2007;66:440-445. doi: 10.1136/ard.2006.058909

Objective: To evaluate the existing evidence on the diagnosis and management of septic arthritis in native joints.

Design: Systematic review.

Data sources: Cochrane Library, Medline, Embase, National Electronic Library for Health, reference lists, national experts. Review methods: Systematic review of the literature with evaluation of the methodological quality of the selected papers using defined criteria set out by the Clinical Effectiveness and Evaluation Unit of the Royal College of Physicians.

Results: 3291 citations were initially identified. Of these, 189 full text articles were identified for potential selection. Following review of these full text articles, 80 articles were found to fulfil the inclusion criteria and were included in the final list. Conclusions were drawn on the diagnosis, investigation and management of septic arthritis.

Discussion: Little good quality evidence exists to guide the diagnosis and management of septic arthritis. Overall, no investigation is more reliable in the diagnosis of septic arthritis than the opinion of an experienced doctor. Aspiration and culture of synovial fluid is crucial to the diagnosis, but measurement of cell count is unhelpful. Antibiotics are clearly required for a prolonged period, but there are no data to indicate by which route or for how long. Key unanswered questions remain surrounding the medical and surgical management of the infected joint.

See end of article for authors' affiliations

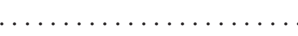

Correspondence to: Dr G Coakley, Queen Elizabeth Hospital, Stadium Road, Woolwich, London UK; gerald.coakley@nhs. net

Accepted 16 December 2006 Published Online First 11 January 2007
7 he presentation of a patient with one or more hot swollen joints is a common medical emergency with a wide differential diagnosis. The most serious of these is septic arthritis. In this systematic review, we define septic arthritis as joint sepsis caused by pathogenic inoculation of the joint by direct or haematogenous routes, rather than an immunological response to pathogens such as that seen in reactive arthritis. Delayed or inadequate treatment of septic arthritis can lead to irreversible joint destruction with subsequent disability, ${ }^{1}$ and in addition there is significant mortality with an estimated case fatality rate of $11 \% .^{2}$ It is therefore vital that the diagnosis is made rapidly and that treatment is started promptly.

The diagnosis of septic arthritis can be difficult to make even in the hands of experienced doctors. However, hot swollen joints frequently present to doctors unfamiliar with the assessment and management of joint disease. Once the diagnosis is made, the optimal management of septic arthritis is a subject of considerable debate. If we are to improve the outcome of septic arthritis, then it is important to develop evidence-based guidelines to aid doctors in both recognising septic arthritis and using rational treatments.

We have performed a systematic review of the literature to identify the best available evidence on the diagnosis and management of hot swollen joints, with a particular emphasis on native joint septic arthritis.

The aims of this review are to:

1. Identify signs, symptoms or risk factors that could aid the correct diagnosis;

2. Identify the evidence underlying the most commonly used management strategies.

\section{METHODS}

We performed a systematic search of the literature using the following databases: Cochrane Library, Medline (1951 to 31 July 2005), Embase (1974 to 31 July 2005) and the National Electronic Library for Health. The search strategies and terms used in the Medline and Embase searches are tabulated in tables 1 and 2. The reference lists of retrieved articles, and of review articles from key authors and journals, were hand searched to confirm the sensitivity of the defined search strategy. Expert members of the group were invited to contribute additional references. Two members of the group independently reviewed the retrieved abstracts. The selection of papers for full text review depended on adherence to defined inclusion and exclusion criteria (table 3 ). We evaluated the methodological quality of the selected papers using defined criteria set out by the Clinical Effectiveness and Evaluation Unit of the Royal College of Physicians (http://rcplondon.ac.uk/college/ceeu/ ceeu_conciseguidance.htm, accessed 4 February 2007)

\section{RESULTS}

The initial search identified 3291 citations. Of these, 189 full text articles were identified for potential selection. After the review of these full text articles, 80 were found to fulfil the inclusion criteria and were included in this systematic review.

Which organisms cause septic arthritis, and What are the risk factors?

All the major surveys of septic arthritis demonstrate that the predominant causative organisms of

Abbreviations: RA, rheumatoid arthritis; WCC, white cell counts 


\begin{tabular}{|ll|}
\hline Table 1 & Medline search strategy \\
\hline 1. & Arthritis-infectious.de \\
2. & Guidelines.pt \\
3. & Meta-analysis articles.pt \\
4. & Randomised controlled trials.pt \\
5. & Controlled clinical trials.pt \\
6. & Evaluation studies.pt \\
7. & 1 and 2 or 3 or 4 or 5 or 6 \\
8. & Drug therapy DT.de \\
9. & Therapy TH.de \\
10. & Diagnosis Dl.de \\
11. & Epidemiology EP.de \\
12. & Microbiology Ml.de \\
13. & Prevention and control PC.de \\
14. & Radiography RA.de \\
15. & Radionuclide inaging Rl.de \\
16. & Surgery SU.de \\
17. & Etiology ET.de \\
18. & Staphylococcal-infections.de \\
19. & Streptococcal-infections.de \\
20. & Pneumococcal-infections.de \\
21. & Neisseria.gonorhhoeae.de \\
22. & Synovial-fluid.de \\
23. & Anti-bacterial-agents.de \\
24. & Joint-prosthesis.de \\
25. & Adrenal-cortex-hormones.de \\
26. & Glucocorticoids.de \\
27. & Arthroscopy.de \\
\hline & \\
\hline
\end{tabular}

septic arthritis are either staphylococci or streptococci. ${ }^{1-8}$ These organisms account for $91 \%$ of cases. ${ }^{2}$

Gram negative organisms are more common in older patients and in those immunocompromised than in young adults. Anaerobic organisms rarely cause septic arthritis, but are more common when there is a history of penetrating trauma. ${ }^{9}$

Risk factors for the development of joint sepsis include:

- rheumatoid arthritis (RA) or osteoarthritis ${ }^{1-5} 10$

- prosthetic joints $^{2}{ }^{3}$

- low socioeconomic status

- intravenous drug abuse ${ }^{24}$

- alcoholism $^{4}$

- diabetes ${ }^{1} 4$

- previous intra-articular corticosteroid injection $^{11}$

- cutaneous ulcers. ${ }^{3}$

A prospective community survey of bacterial arthritis found that a number of factors seem to be associated with a poor prognosis. These include older age, pre-existing joint disease and the presence of synthetic material within the joint. ${ }^{12}$

\section{Symptoms and signs suggestive of septic arthritis}

Septic arthritis typically presents as a hot, swollen, tender joint or joints with a reduced range of movement. ${ }^{2}{ }^{10}$ Symptoms are usually present for $<2$ weeks at presentation, but significant delays may occur, particularly with low virulence organisms, tuberculosis and prosthesis infection. ${ }^{2}{ }^{13}$ Large joints are more commonly reported than small joints and in up to $60 \%$ of cases the joints affected are the hip or the knee. ${ }^{311}{ }^{14}$ Where there is pre-existing inflammatory joint disease, such as RA, the symptoms in the affected joint, or joints, are out of proportion to the disease activity detected in other joints.

In up to $22 \%$ of cases, more than one joint is affected and therefore an oligoarticular or polyarticular presentation does not exclude the diagnosis of sepsis. ${ }^{35}$ Gonococci and meningococci have an increased tendency, compared with other causative organisms, to affect more than one joint. ${ }^{15}$ The
Table 2 EMBASE search strategy

\begin{tabular}{ll}
\hline 1. & Infectious-arthritis.de \\
2. & Diagnosis DI.de \\
3. & Disease management DM.de \\
4. & Drug therapy DT.de \\
5. & Epidemiology EP.de \\
6. & Etiology ET.de \\
7. & Prevention PC.de \\
8. & Surgery SU.de \\
9. & Therapy TH.de \\
10. & Practice-Guideline.de. \\
11. & Antibiotic-agent.de \\
12. & Randomised controlled trials \\
13. & Meta analysis \\
14. & Staphylococcus-aureus.de. \\
15. & Streptococcus-infection .de. \\
\hline
\end{tabular}

presence of fever is not a reliable indicator of an infected joint. ${ }^{12513}$

\section{Investigation of suspected septic arthritis Investigation of synovial fluid}

Prompt microscopic analysis and culture of synovial fluid are fundamental diagnostic tools in the evaluation of possible joint sepsis, enabling the diagnoses of both sepsis and crystal induced arthritis to be confirmed rapidly. ${ }^{16}$ Culture is more sensitive than microscopy alone, as synovial fluid Gram staining is positive in only $50 \%$ of cases. ${ }^{1}$

Some have argued that the total and differential white cell counts (WCCs) in synovial fluid can provide a simple way of distinguishing between infected and non-infected joints. ${ }^{17} \mathrm{We}$ examined the literature for evidence that the synovial fluid WCC might be helpful in further aiding the diagnostic process.

Coutlakis demonstrated in a retrospective study that, out of 202 patients, those with synovial fluid WCCs of $>50000 / \mathrm{mm}^{3}$ and $>100000 / \mathrm{mm}^{3}$ had a diagnosis of septic arthritis in $47 \%$ and $77 \%$ of patients, respectively. The conclusion was that those patients with a synovial WCC of $<50000 / \mathrm{mm}^{3}$ had a reduced likelihood of infection, but it was not excluded. ${ }^{17}$ Soderquist et al looked at a number of serological (WCC, tumour necrosis factor $\alpha$, interleukin 6 and interleukin 8) and synovial (WCC, glucose) markers and found that none of them had sufficient sensitivity, specificity or predictive value to be of use in discriminating between infective and non-infective arthritis. ${ }^{18}$ We conclude that the synovial fluid WCC is not sufficiently reliable a measure to exclude or confirm a diagnosis of septic arthritis.

Although much has been written on the value of lactic acid levels in diagnosing septic joints, only three published studies fitted the criteria for inclusion in our search. ${ }^{19-21}$ Lactic acid levels rise in the synovial fluid with sepsis, but they also rise in joint inflammation of any cause. It has not been established that lactic acid levels have any discriminant value.

\section{Handling of synovial fluid specimens}

The cornerstone of rapid and reliable confirmation of the diagnosis of suspected septic arthritis is microbiological examination of the synovial fluid. The sensitivity of detection of causative organisms is, however, often disappointing. Neisserial organisms are particularly fastidious, and this could explain their low yields from culture in case series. No evidence was found to suggest how the handling of samples from suspected Neisserial arthritis should differ, but inoculation into Neisserial-specific media should increase the yield.

It is generally accepted that samples of freshly aspirated joint fluid should be sent to the laboratory for immediate analysis, 
Table 3 Inclusion and exclusion criteria

\begin{tabular}{ll}
\hline $\begin{array}{l}\text { Inclusion } \\
\text { criteria }\end{array}$ & $\begin{array}{l}\text { Exclusion } \\
\text { criteria }\end{array}$ \\
\hline Randomised controlled trials & Paediatric studies (age $<16$ years) \\
Controlled clinical trials & Animal studies \\
$\begin{array}{l}\text { Prospective observational } \\
\text { studies }\end{array}$ & Case reports \\
$\begin{array}{l}\text { Retrospective observational } \\
\text { studies }\end{array}$ & Case series of $<40$ patients \\
Guidelines & Review articles \\
Meta-analyses & $\begin{array}{l}\text { Studies on reactive arthritis } \\
\text { Case series including }\end{array}$ \\
40 or more cases & $\begin{array}{l}\text { Studies on infection in other musculoskeletal } \\
\text { sites (eg, osteomyelitis, tenosynovitis) } \\
\text { Chronic sepsis } \\
\text { Spinal infection and back pain } \\
\text { Osteoarthritis } \\
\text { Gout } \\
\text { Management of septic arthritis beyond the } \\
\text { first } 6 \text { weeks }\end{array}$ \\
\hline
\end{tabular}

although no studies were found to support the importance of these factors. Some investigators, however, have examined whether the technique used to isolate and culture bacteria from joint fluid can be modified to increase the yield.

An analysis of 47 episodes of bacterial arthritis showed that one third of specimens from patients not receiving antibiotics, and half of those from patients receiving antibiotics, were positive after inoculation of synovial fluid into blood culture bottles, despite negative results from culture on conventional solid media. The opinion of the authors was that these extra organisms were pathogenic and not contaminants. The authors suggested that it should be routine practice to inoculate blood culture bottles in the laboratory to increase the yield of fastidious organisms, in addition to direct culture on agar plates. ${ }^{22}$ Inoculation of blood culture bottles at the bedside was not recommended.

Subsequent studies have confirmed that both inoculation into culture bottles and the use of lysis centrifugation tubes in the laboratory are superior to conventional agar plate methods for the detection of clinically significant micro-organisms. ${ }^{23} \mathrm{~A}$ subsequent study, however, suggested that the choice of culture method was less critical, if the sample was obtained and preserved with caution, with attention to aseptic technique throughout and immediate transport to the laboratory. ${ }^{24}$

The value of PCR in the diagnosis of joint infection Two adequately powered studies examined whether investigation of synovial fluid using the PCR assay may allow more rapid or accurate diagnosis. The results indicated that, in the standard laboratory setting, PCR did not offer any advantage over bacterial culture in the microbiological diagnosis of staphylococcal or streptococcal joint infection. ${ }^{25}{ }^{26}$ There are specific organisms, such as Borrelia species, which have seldom been cultured from joint specimens, and in these situations PCR may be a useful complementary tool.

\section{Other investigations}

Blood cultures may identify the causative organism even where synovial fluid culture is unrewarding. ' The serum WCC, erythrocyte sedimentation rate and $\mathrm{C}$ reactive protein are helpful in monitoring response to treatment. Many studies have shown that the absence of a raised serum WCC, erythrocyte sedimentation rate or $\mathrm{C}$ reactive protein does not exclude the diagnosis of sepsis. ${ }^{2}$

\section{Imaging}

There are no robust studies prospectively investigating the optimal imaging methods to be used in the diagnosis of septic arthritis. As with many studies of septic arthritis, the problems of ascertainment bias and the lack of a suitable gold standard hinder evaluation. Notwithstanding this, some conclusions may be drawn.

Scintigraphy performs well for distinguishing sepsis from osteoarthrosis, but cannot distinguish effectively between sepsis and other causes of joint inflammation. ${ }^{27} \mathrm{~A}$ study on MRI findings described appearances that would correlate most accurately with the presence of infection, but no sterile inflammatory controls were included in the study design. ${ }^{28} \mathrm{~A}$ recent expert review concluded that no radiological technique is currently sufficiently sensitive or specific to be diagnostically useful in suspected septic arthritis. Plain radiography, although not useful for imaging of the joint, may be performed routinely to exclude underlying osteomyelitis. ${ }^{29}$

\section{Management of septic arthritis}

\section{Antibiotic treatment for septic arthritis}

Prompt treatment with antibiotics together with removal of any purulent material is the mainstay of treatment for septic arthritis. There is little evidence on which to base the choice and duration of antibiotic treatment, and we found no randomised, controlled trials. One systematic review and meta-analysis of antibiotic treatment for joint infections showed no advantage in clinical or bacteriological efficacy of one antibiotic regimen over another. ${ }^{30}$ Currently, the choice of antibiotic is based on the likelihood of the organism involved,

Table 4 Suggested empirical antibiotic treatment of suspected septic arthritis

\begin{tabular}{ll}
\hline Patient group & Antibiotic choice \\
\hline No risk factors for atypical & Flucloxacillin $2 \mathrm{~g}$ qds IV. Local \\
organisms & policy may be to add fusidic acid \\
& $500 \mathrm{mg}$ tds po, or gentamicin IV. \\
& If penicillin is allergic, clindamycin \\
& $450-600 \mathrm{mg}$ qds, or 2nd or 3rd \\
& generation cephalosporin may be \\
& given
\end{tabular}

High risk of Gram negative sepsis (elderly, frail, recurrent UTI, recent abdominal surgery)

2nd or 3rd generation cephalosporin (eg, cefuroxime $1.5 \mathrm{~g} \mathrm{tds}$ ). Local policy may be to add flucloxacillin. Discuss allergic patients with microbiology-Gram stain may influence the choice of antibiotic

MRSA risk (known MRSA, recent inpatient, nursing home resident, leg ulcers or catheters, or other risk factors determined locally)

\section{Suspected gonococcus or} meningococcus

Vancomycin plus 2nd or 3rd generation cephalosporin depending on local policy/ resistance

IV drug users

Discuss with microbiologist

ITU patients, known colonisation of other organs (eg, cystic fibrosis)

ITU, intensive therapy unit; IV, intravenous; MRSA, methicillin-resistant Staphylococcus aureus; qds, four times in a day; UTI, urinary tract infection; tds, thrice a day.

Antibiotic choice will need to be modified in the light of results of Gram stain and culture. It should also be reviewed locally by microbiology departments. 


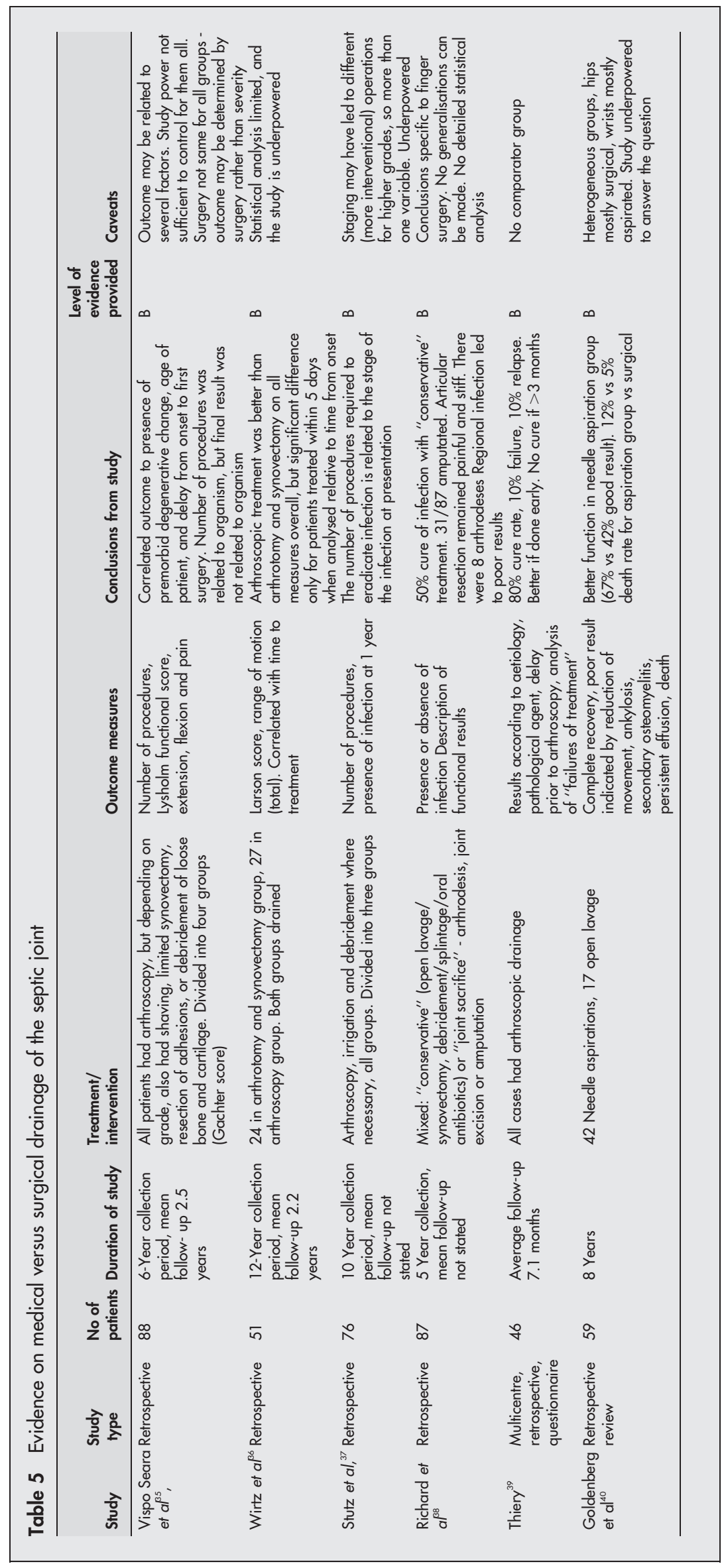




\section{What is already known on this topic}

- Hot swollen joints are a common emergency, the most serious cause of which is septic arthritis.

- Patients often present to doctors who are not experienced in musculoskeletal medicine.

- The diagnosis can be difficult as, even among specialists, there can be a lack of consensus on the key management issues.

modified by the results of Gram staining and culture. A summary of suggested empirical antibiotic treatment is presented in table $4 .{ }^{29}$

\section{Joint drainage and surgical options}

Successful treatment of a septic native joint includes removal of purulent material from the joint space. This can be achieved either surgically or through closed needle aspiration. There is controversy over which mode of drainage should be employed.

Six papers were retrieved which pertained to joint drainage and surgical management of the septic joint. Of these, only one compared needle aspiration and surgical joint drainage, but this was a retrospective analysis of the case records of proven cases of bacterial arthritis. ${ }^{31}$ The results suggested that needle aspiration appeared, in general, to be preferable to surgical treatment as an initial mode of treatment of joint sepsis, although the results did not reach statistical significance (and there was a trend towards higher mortality in the needle aspiration group). The authors suggested that in certain circumstances, such as hip sepsis, surgical management would be advocated. The study had many limitations, including a small number of cases, retrospective design, lack of randomisation to treatment and case matching.

The other five papers retrieved were also retrospective observational studies of case series of joint sepsis treated by arthroscopy and washout with or without synovectomy, arthrotomy or debridement. ${ }^{32-35}$ None of these studies yielded useful information on whether conservative or surgical measures should be employed (table 5 ).

\section{DISCUSSION}

\section{Methodology}

In performing this review, we followed an explicit and reproducible search strategy. We structured the search by defining key questions that needed to be answered.

\section{Limitations on the quality of evidence obtained}

There was a striking lack of robust clinical evidence on the subject of septic arthritis. We found no randomised controlled trials. Many of the papers were retrospective, descriptive studies, and as a result carried the risk of distortion through ascertainment bias. Some papers admitted the possibility that cases of septic arthritis may have been missed through errors in diagnostic classification or failure to obtain case records. On the whole, however, the papers included in this review had used methods that minimised such risks, while admitting that $100 \%$ case inclusion could not be guaranteed. The quality of evidence available to us limits the strength of the conclusions that we are able to draw.

\section{Principal findings}

Despite being an important clinical presentation with significant potential morbidity and mortality, there is little highquality evidence to guide practitioners in the diagnosis of septic arthritis. Likewise, after the diagnosis, there is scant evidence to guide the optimum medical and surgical management of this condition.

\section{What this study adds}

- This systematic review demonstrates that the overall impression of a clinician experienced in assessing joint disease is the gold standard for diagnosing septic arthritis.

- Demographic factors can predict atypical organisms. Although there have been no randomised controlled trials, the outcome appears similar, irrespective of whether medical or arthroscopic approaches to treatment are used.

What is clear is that septic arthritis almost invariably presents over a period of up to 2 weeks as one or more hot, painful, swollen and restricted joints, and in this circumstance sepsis should be assumed until proved otherwise. The absence of a fever or raised white cell count cannot reliably exclude the diagnosis, nor can a negative synovial fluid culture. There is no gold standard for the diagnosis of septic arthritis, save that the overall judgement of an experienced clinician has proved superior to any laboratory or radiological investigation. ${ }^{14}$ If the diagnosis is suspected, then advice from a musculoskeletal specialist should be sought at the earliest opportunity.

Risk factors (old age, prior hospitalisation, trauma or leg ulceration) may be predictive of the likely infective organism, so a careful history is useful in guiding antibiotic choice. Regarding laboratory tests, inoculation of synovial fluid into blood culture bottles or lysis centrifugation techniques in the microbiology laboratory seems to increase the diagnostic yield. No other serological, synovial fluid or radiological test has been shown to be adequately discriminatory. Antibiotic treatment is clearly required, although which to choose, by which route and for how long remain guided by precedent rather than evidence.

\section{Key unanswered questions}

These include antibiotic choice, route of administration and duration in the treatment of joint sepsis. There is also no evidence to settle the debate over whether closed aspiration or open arthroscopy/arthrotomy is superior in removing pus from an infected joint. There is a need for randomised controlled trials to address these questions. Logistic reasons would make such trials extremely challenging, perhaps illustrating some of the limitations of evidence-based medicine.

\section{ACKNOWLEDGEMENTS}

This systematic review was carried out to provide information for guidelines on management of hot swollen joints commissioned by the British Society for Rheumatology and the British Health Professionals in Rheumatology. We acknowledge their support, and that of Arthritis Care. We are also grateful to the library staff at Queen Elizabeth Hospital, Woolwich, for help with performing the systematic review and retrieving references.

\section{Authors' affiliations}

C J Mathews, Queen Elizabeth Hospital, Woolwich, London, UK G Kingsley, University Hospital Lewisham, Kings College London, Weston Education Centre, Cutcombe Road, London, SE5 9RJ, UK

M Field, Centre for Rheumatic Diseases, Glasgow Royal Infirmary, Glasgow, UK

A Jones, Rheumatology Unit, City Hospital Trust, Nottingham, UK V C Weston, Department of Microbiology, University Hospital NHS Trust, Queen's Medical Centre, Nottingham, UK

M Phillips, King's College Hospital, London, UK

D Walker, Musculoskeletal Unit, Freeman Hospital, Newcastle upon Tyne, UK

G Coakley, Queen Elizabeth Hospital, Stadium Road, Woolwich, London SE18 4QH, UK 
Competing interests: None.

Ethical approval was not required for this review.

Contribution: CJM conducted the literature search. GC and CJM independently reviewed the abstracts retrieved from the search and categorised for inclusion or exclusion according to the defined criteria. MF, AJ, VCW and MP evaluated the quality of the papers according to their specialist interests. DW and GK provided expert advice on the conclusions drawn from the papers. All authors and contributors reviewed and advised on the final manuscript.

\section{REFERENCES}

1 Weston VC, Jones AC, Bradbury N, Fawthrop F, Doherty M. Clinical features and outcome of septic arthritis in a single UK health district 1982-1991. Ann Rheum Dis 1999:58:214-9.

2 Gupta MN, Sturrock RD, Field M. A prospective 2-year study of 75 patients with adult-onset septic arthritis. Rheumatology (Oxford) 2001;40:24-30.

3 Kaandorp CJ, Dinant HJ, van de Laar MA, Moens HJ, Prins AP, Dijkmans BA. Incidence and sources of native and prosthetic joint infection: a community based prospective survey. Ann Rheum Dis 1997;56:470-5.

4 Sharp JT, Lidsky MD, Duffy J, Duncan MW. Infectious arthritis. Arch Intern Med 1979; 139:1125-30.

5 Dubost JJ, Fis I, Denis P, Lopitaux R, Soubrier M, Ristori JM, et al. Polyarticular septic arthritis. Medicine (Baltimore) 1993;72:296-310.

6 Ryan MJ, Kavanagh R, Wall PG, Hazleman BL. Bacterial joint infections in England and Wales: analysis of bacterial isolates over a four year period. Br J Rheumatol 1997:36:370-3.

7 Dubost JJ, Soubrier M, De Champs C, Ristori JM, Bussiere JL, Sauvezie B. No changes in the distribution of organisms responsible for septic arthritis over a 20 year period. Ann Rheum Dis 2002;61:267-9.

8 Goldenberg DL, Cohen AS. Acute infectious arthritis. A review of patients with nongonococcal joint infections (with emphasis on therapy and prognosis). Am J Med 1976;60:369-77.

9 Brook I, Frazier EH. Anaerobic osteomyelitis and arthritis in a military hospital: a 10-year experience. Am J Med 1993;94:21-8.

10 Ispahani P, Weston VC, Turner DP, Donald FE. Septic arthritis due to Streptococcus pneumoniae in Nottingham, United Kingdom, 1985-1998. Clin Infect Dis 1999;29:1450-4.

11 Meijers KA, Dijkmans BA, Hermans J, van den Broek PJ, Cats A. Nongonococcal infectious arthritis: a retrospective study. J Infect 1987;14:13-20.

12 Kaandorp CJ, Krijnen P, Moens HJ, Habbema JD, van Schaardenburg D. The outcome of bacterial arthritis: a prospective community-based study. Arthritis Rheum 1997;40:884-92.

13 Gupta MN, Sturrock RD, Field M. Prospective comparative study of patients with culture proven and high suspicion of adult onset septic arthritis. Ann Rheum Dis 2003;62:327-31

14 Rosenthal J, Bole GG, Robinson WD. Acute nongonococcal infectious arthritis Evaluation of risk factors, therapy, and outcome. Arthritis Rheum 1980;23:889-97.

15 Manshady BM, Thompson GR, Weiss JJ. Septic arthritis in a general hospital 1966-1977. J Rheumatol 1980;7:523-30.

16 Swan A, Amer H, Dieppe P. The value of synovial fluid assays in the diagnosis of joint disease: a literature survey. Ann Rheum Dis 2002;61:493-8.

17 Coutlakis PJ. In:, Roberts WN, Wise CM, eds. Another look at synovial fluid leukocytosis and infection. J Clin Rheumatol 2002;8:67-71.
18 Soderquist B, Jones I, Fredlund H, Vikerfors T. Bacterial or crystal-associated arthritis? Discriminating ability of serum inflammatory markers. Scand J Infect Dis 1998;30:591-6.

19 Kortekangas P, Peltola O, Toivanen A, Aro HT. Synovial fluid L-lactic acid in acute arthritis of the adult knee joint. Scand J Rheumatol 1995;24:98-101.

20 Brook I, Reza MJ, Bricknell KS, Blvestone R, Finegold SM. Abnormalities in synovial fluid of patients with septic arthritis detected by gas-liquid chromatography. Ann Rheum Dis 1980;39:168-72.

21 Brook I, Reza MJ, Bricknell KS, Bluestone R, Finegold SM. Synovial fluid lactic acid. A diagnostic aid in septic arthritis. Arthritis Rheum 1978;21:774-9.

22 von Essen R, Holtta A. Improved method of isolating bacteria from joint fluids by the use of blood culture bottles. Ann Rheum Dis 1986;45:454-7.

23 Yagupsky P, Press J. Use of the isolator 1.5 microbial tube for culture of synovial fluid from patients with septic arthritis. J Clin Microbiol 1997:35:2410-12.

24 Kortekangas P, Aro HT, Lehtonen OP. Synovial fluid culture and blood culture in acute arthritis. A multi-case report of 90 patients. Scand J Rheumatol 1995;24:44-7.

25 Jalava J, Skurnik M, Toivanen A, Toivanen P, Eerola E. Bacterial PCR in the diagnosis of joint infection. Ann Rheum Dis 2001;60:287-9.

26 Tarkin IS, Henry TJ, Fey PI, Iwen PC, Hinrichs SH, Garvin KL. PCR rapidly detects methicillin-resistant staphylococci periprosthetic infection. Clin Orthop Relat Res 2003:89-94.

27 Niihof MW, Oyen WJ, van Kampen A, Claessens RA, van der Meer JW, Corstens FH. Evaluation of infections of the locomotor system with indium-111labeled human IgG scintigraphy. J Nucl Med 1997;38:1300-5.

28 Karchevsky M, Schweitzer ME, Morrison WB, Parellada JA. MRI findings of septic arthritis and associated osteomyelitis in adults. Am J Roentgenol 2004:182:119-22.

29 Coakley G, Mathews C, Field M, Jones A, Kingsley G, Phillips $M$, et al. BSR \& BHPR, BOA, RCGP and BSAC guidelines for the management of the hot swollen joint in adults. Rheumatology (Oxford) 2006;45:1039-41.

30 Stengel D, Bauwens K, Sehouli J, Ekkernkamp A, Porzsolt F. Systematic review and meta-analysis of antibiotic therapy for bone and joint infections. Lancet Infect Dis $2001 ; 1: 175-88$.

31 Goldenberg DL, Brandt KD, Cohen AS, Cathcart ES. Treatment of septic arthritis: comparison of needle aspiration and surgery as initial modes of joint drainage. Arthritis Rheum 1975;18:83-90.

32 Richard JC, Vilain R. Acute septic arthritis of the fingers. A clinical study of 87 cases. Ann Chir Main 1982;1:214-20.

33 Wirtz DC, Marth M, Miltner O, Schneider U, Zilkens KW. Septic arthritis of the knee in adults: treatment by arthroscopy or arthrotomy. Int Orthop 2001;25:239-41.

34 Stutz G, Kuster MS, Kleinstuck F, Gachter A. Arthroscopic management of septic arthritis: stages of infection and results. Knee Surg Sports Traumatol Arthrosc 2000;8:270-4

35 Vispo Seara JL, Barthel T, Schmitz H, Eulert J. Arthroscopic treatment of septic joints: prognostic factors. Arch Orthop Trauma Surg 2002;122:204-11.

36 Wirtz DC, Marth M, Miltner O, Schneider U, Zilkens KW. Septic arthritis of the knee in adults: treated by arthroscopy or arthrotomy. Intl Orthop 2001;25:239-41.

37 Stutz G, Kuster MS, Kleinstuck F, Gächter A. Arthroscopic management of septic arthritis: stages of infection and results Knee Surg, Sports Traumatol, Arthrosc 2000;8:270-4

38 Richard JC, Vilain R. Acute septic arthritis of the fingers: a clinical study of 87 cases. Ann Chir Main 1982;1:3214-220.

39 Thiery JA. Arthroscopic drainage in septic arthritides of the knee: a multicenter study. Arthroscopy 1989;5:65-9.

40 Goldenberg DL, Brandt KD, Cohen AS, Cathcart ES. Treatment of septic arthritis: comparison of needle aspiration and surgery as initial modes of joint drainage Arthritis and Rheum 1975;18:83-9. 